\title{
硅碱钙石微晶玻璃的析晶特性及其增韧机理研究
}

\author{
李要辉, 王晋珍, 黄幼榕
}

(中国建筑材料科学研究总院玻璃科学研究院, 北京 100024)

摘 要: 通过调整热处理工艺制备了不同结构的 $\mathrm{R}_{2} \mathrm{O}-\mathrm{CaO}-\mathrm{SiO}_{2}-\mathrm{F}$ 系硅碱䥻石微晶玻璃, 分析了析晶特性和力学性 能, 重点研究以硅碱钻石为主晶微晶玻璃的裂纹扩展机制及增韧机理。结果表明, 基础玻璃在低温处理时首先析出 $\mathrm{CaF}_{2}$ 微晶作为异质晶核，一步法处理后得到岛屿状硬硅钻石/硅碱钻石复相微晶玻璃，两步法处理后得到了力学性 能优异、具有板条交错结构的硅碱钻石微晶玻璃。研究发现，硅碱钙石微晶玻璃的裂纹扩展受到周围板条晶体取向 及晶界影响, 存在穿晶断裂和沿晶断裂两种模式扩展, 呈现出随机取向的折线或台阶状裂纹路径。其中具有一定长 径比的板条状硅碱钻石具有较好的桥联作用，承载补强效果显著，此外硅碱钻石晶体与玻璃相之间存在的残余应 力，有利于缓解裂纹应力集中以及增加裂纹扩展能。即，硅碱钻石微晶玻璃优异的力学性能是各种增强和增㓞机制 综合作用的结果。

关 键 词: $\mathrm{R}_{2} \mathrm{O}-\mathrm{CaO}-\mathrm{SiO}_{2}-\mathrm{F}$ 系微晶玻璃; 硅碱钻石; 裂纹扩展; 增韧机理

中图分类号: TQ174 文献标识码: A

\section{Characterization and Toughening Mechanism of Crystallization of Canasite Glass-ceramics}

\author{
LI Yao-Hui, WANG Jin-Zhen, HUANG You-Rong \\ (Glass Science Institute, China Building Material Academy, Beijing 100024, China)
}

\begin{abstract}
The $\mathrm{R}_{2} \mathrm{O}-\mathrm{CaO}-\mathrm{SiO}_{2}-\mathrm{F}$ system glass-ceramics were prepared through different heat treatment schedules. The crystallization, microstructure and mechanical properties, especially the crack propagation and toughening mechanism of the canasite-based glass-ceramics, were studied. The results show that $\mathrm{CaF}_{2}$ crystallites precipitate firstly during heat treatment at low temperature. After one-step heat treatment block-like xonotlite/canasite complex phases can be obtained. And after two-step heat treatment, the canasite-based glass-ceramics, composed of particularly interlocking blade-like crystals, are obtained with excellent mechanical properties. The micrographs suggest the orientation and interface of canasite crystals radically change the crack propagation, showing intercrystalline and transcrystalline cracks forming random-step or fold-like line. Based on these results, the blade-like canasite crystals at certain slenderness ratios, display favorable anti-cracking effect because of their bridging toughen mechanism. In addition, the residual stress between matrix and crystals also benefits relaxation of stress and deflection of crack. So, the excellent mechanical properties of the as-prepared glass-ceramics are resulting from multiple toughening mechanisms.
\end{abstract}

Key words: $\mathrm{R}_{2} \mathrm{O}-\mathrm{CaO}-\mathrm{SiO}_{2}$-F glass-ceramics; canasite; crack propagation; toughening mechanism

$\mathrm{R}_{2} \mathrm{O}-\mathrm{CaO}-\mathrm{SiO}_{2}-\mathrm{F}$ 系统( $\mathrm{RCSF}$ )玻璃通过受控晶化 可获得以硅碱钙石为主晶相的高强韧微晶玻璃 ${ }^{[1-2]}$ 。
Beall 等 ${ }^{[3-4]}$ 研究发现该玻璃在 $700 \sim 850^{\circ} \mathrm{C}$ 保温处理 $1 \mathrm{~h}$ 可以通过非均匀成核诱发氟硅碱钙石的核化, 
玻璃内部形成粒度约为 $0.5 \mu \mathrm{m}$ 的 $\mathrm{CaF}_{2}$ 微晶作为异 相晶核, 硅碱钻石原位快速生长形成致密的板条交 错微观结构。由于该微晶玻璃含有大量各向异性晶 体及相互交错的特殊微观结构, 其断裂韧性可达 4.8 5.2 $\mathrm{MPa} \cdot \mathrm{m}^{1 / 2}$, 是目前韧性较高的一种微晶玻 璃。同时由于该玻璃熔制温度较低、料性好易成形, 其中以 $\mathrm{F}$ 为晶核剂时通过核化-晶化两步法热处理 可得到具有半透明玉质特征的微晶玻璃, 目前作为 一种新型的建筑装饰材料颇受欢迎。此外, 由于它 具有高强、高韧及耐磨等性能, 在生物医用领域 ${ }^{[5-7]}$ 及其他复合材料 ${ }^{[8]}$ 中也有一些应用研究。

由于该系统微晶玻璃的析晶过程及组织结构复 杂多变, 关于其组成和析晶过程的研究较多。美国康 宁公司 ${ }^{[3]}$ 最早发现了该材料的独特性能, 英国谢菲尔 德大学围绕该玻璃在生物材料中的应用, 针对其形成 机理 ${ }^{[9-10]}$ 、成分变化 ${ }^{[11]}$ 和制备条件 ${ }^{[12-13]}$ 等对组织结构 和性能的影响进行了全面分析, 并利用 TEM 等先进 手段对玻璃内部分相、 $\mathrm{CaF}_{2}$ 微晶形核和 Canasite 晶体 生长进行了深入研究。国内也有多位研究者围绕 $\mathrm{CaO} 、 \mathrm{~K}_{2} \mathrm{O} 、 \mathrm{Al}_{2} \mathrm{O}_{3} 、 \mathrm{~F}$ 和 $\mathrm{SiO}_{2}$ 等成分 ${ }^{[14-20]}$ 对硅碱钻石 微晶玻璃结构及析晶的影响开展了工作 ${ }^{[21]}$, 部分研究 还涉及到微晶玻璃的着色 ${ }^{[22-23]}$ 及化学稳定性 ${ }^{[24]}$, 使得 该玻璃体系的技术数据更加丰富。

作者研究发现, 不同的晶相类型以及它们的微 观结构对性能的影响是非常明显的, 不同形核温度/ 形核时间、析晶温度/析晶时间组合引起的硅碱钙石 组织结构的微小变化就会带来力学性能的巨大差异, 这些不确定的因素都为该材料的设计及应用带来了 不便。鉴于目前关于硅碱钻石微晶玻璃组织结构与力 学性能的关系研究较少, 尤其是在微观尺度上其裂纹 扩展模式和增韧机理尚不清晰, 因此本工作在研究该 硅碱钙石微晶玻璃组织结构和性能基础上, 深入讨论 其微观结构与裂纹扩展机制及增韧机理的关系。

\section{1 样品制备与测试方法}

实验在已有 “玉石型” 建筑装饰微晶玻璃基础 上，选取的基础玻璃成分为: $\mathrm{SiO}_{2} 60 \% \sim 65 \%, \mathrm{CaO}$ $15 \% \sim 18 \%, \mathrm{Na}_{2} \mathrm{O}+\mathrm{K}_{2} \mathrm{O} 12 \% \sim 15 \%, \mathrm{Al}_{2} \mathrm{O}_{3} 2 \% \sim 3 \%, \mathrm{~F}$ 2\% 4\%, 其他 2\% 5\%。选取化学纯粉末试剂为基础 玻璃制备原料, 其中碱金属、碱土金属氧化物以碳 酸盐形式引入, $\mathrm{F}$ 元素以 $\mathrm{CaF}_{2}$ 形式引入, 其余为氧 化物, 经精确称量后将配合料充分研磨混匀, 放入 氧化铝坩埚, 在马弗炉中 $1450 \sim 1480^{\circ} \mathrm{C}$ 熔化 1 2 h 制 得均匀澄清的玻璃熔体。将熔融好的玻璃液倾倒在 预热的铁板上, 经退火降至室温, 得到基础玻璃。热 处理后制成微晶玻璃样块, 其中一步法和两步法热
处理工艺制度如图 1 所示。

利用 NETZSCH 公司 STA 409 C 同步热分析仪 对基础玻璃粉进行差热分析, 升温速率为 $10^{\circ} \mathrm{C} / \mathrm{min}$, 温度范围为 $30 \sim 900^{\circ} \mathrm{C}$ 。利用 Rigaku D/Max-2500 X 射线, 衍射仪进行物相测定, 加速电压和电流分别 为 $40 \mathrm{kV}$ 和 $200 \mathrm{~mA}$, 扫描速度为 $6 \%$ min, 步长 $0.02^{\circ}$, 铜靶。微晶玻璃样品经研磨抛光后, 在浓度为 $2 \%$ 的 $\mathrm{HF}$ 酸溶液中侵蚀 $60 \mathrm{~s}$, 喷金处理后, 采用热场发射 LEO-1530 型扫描电镜观察其组织形貌。基础玻璃和 微晶玻璃的样品表面抛光处理后, 利用美国 MTS 公司的纳米压痕仪(NANO Indenter)按照 CSM(连续 刚度)法测量维氏硬度 $H$ 和弹性模量 $E$ 。并分别按照 $\mathrm{JC} / \mathrm{T} 872-2000$ 和 JC/T259-93 标准制备样品测试其弯 曲强度和冲击韧性。利用 HXD-1000TMC 显微硬度 计, 选择 $4.9 \mathrm{~N}$ 压力在抛光样品表面预制压痕, 保压 时间 $10 \mathrm{~s}$, 同时利用 SEM 观察压痕形貌及裂纹扩展, 根据载荷和裂纹长度测量结果利用压痕法(IM 法)计 算材料的断裂韧性 $K_{\mathrm{IC}}$ ，计算公式如下:

$$
K_{\mathrm{IC}}=0.028 \times H a^{1 / 2}\left(\frac{E}{H}\right)^{1 / 2}\left(\frac{c}{a}\right)^{-3 / 2}
$$

式中 $a$ 为压痕凹坑对角线半长度; $c$ 为裂纹对称扩展 的半长度。

\section{2 结果与讨论}

\subsection{RCSF 玻璃的析晶过程研究}

图 2 为四炉次基础玻璃的 DSC 曲线, 由图中可 见该玻璃的玻璃化转变温度 $T_{\mathrm{g}}$ 介于 $550 \sim 560^{\circ} \mathrm{C}$, 中 温 665 680 ${ }^{\circ} \mathrm{C}$ 对应于分相形核阶段 $\mathrm{CaF}_{2}$ 微晶的形成, 而 $800 \sim 815^{\circ} \mathrm{C}$ 范围内的放热峰对应于硅碱钙石晶相 的析出。综合对比四条曲线, 可以认为不同批次玻 璃的热特性是基本一致的，可以排除因基础玻璃差 异带来的影响。根据 DSC 测试结果, 一步法热处理 温度区间选择为 $700 \sim 850^{\circ} \mathrm{C}$, 温度间隔为 $50^{\circ} \mathrm{C}$; 两 步法热处理制度为在 $650 \sim 700^{\circ} \mathrm{C}$ 核化处理 $1.5 \sim 2 \mathrm{~h}$, 然后升温至 $850^{\circ} \mathrm{C}$ 晶化处理 $1 \mathrm{~h}$, 如图 1 所示。

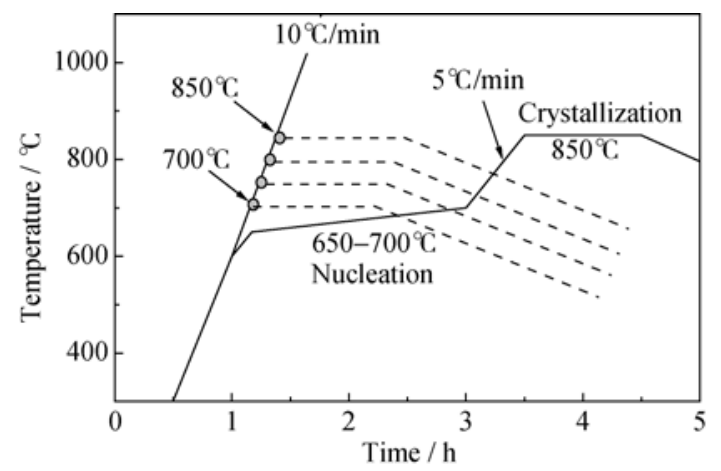

图 1 基础玻璃不同热处理制度曲线

Fig. 1 Diagram of the heat treatment schedule 


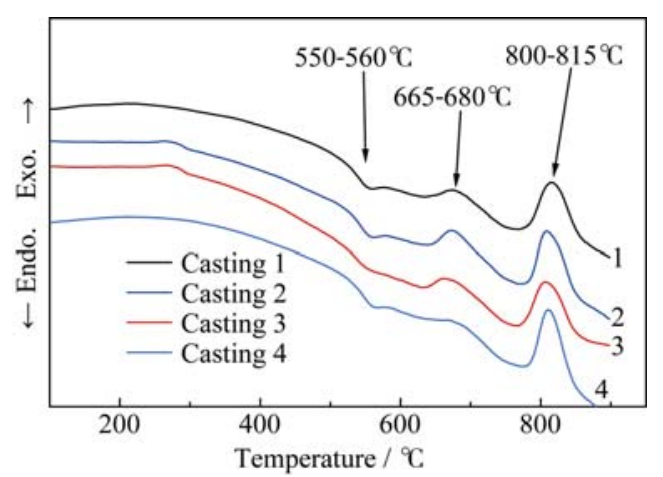

图 2 不同炉次基础玻璃的 DSC 曲线

Fig. 2 DSC curves of different RCSF casting glasses

图 3 为一步法不同温度保温 $1 \mathrm{~h}$ 热处理后的 XRD 图谱, 在 $20^{\circ} \sim 35^{\circ}$ 范围内均可见明显的非晶态 玻璃相衍射带, 但 $700^{\circ} \mathrm{C}$ 处理后就可见微弱析晶衍 射峰, 其中 $28^{\circ} 、 47^{\circ} 、 56^{\circ}$ 为 $\mathrm{CaF}_{2}$ 晶相的典型衍射 峰, 对应于基础玻璃外观乳浊失透现象。热处理温 度升高至 $800^{\circ} \mathrm{C}$, 出现对应于硬硅钙石晶相 (Xonotlite PDF 23-0125)的衍射峰, 但其衍射峰强度 较弱, 晶体含量较低。在 $850^{\circ} \mathrm{C}$ 保温处理 $1 \mathrm{~h}$ 后, 除 会在此基础上形核长大, 随着热处理温度升高部分 上述两种晶相外, 在低角度范围内出现了一系列对 应于硅碱钻石(Canasite-A PDF 45-1398)晶相的衍射 峰。可见, RCSF 玻璃在低温析晶处理会形成大量晶 粒细小的球形 $\mathrm{CaF}_{2}$ 微晶, 硬硅钻石或硅碱钙石晶体

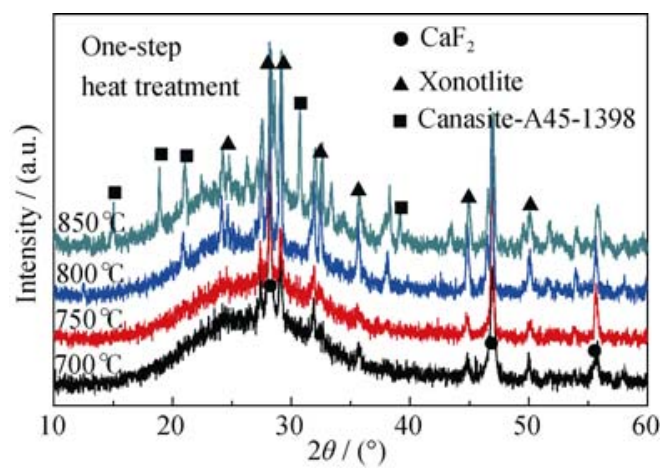

图 3 不同温度一步法热处理后样品的 XRD 图谱

Fig. 3 XRD patterns of glass-ceramics after one-step heat treatment
硬硅钙石转变为硅碱钙石晶体，这与文献报道是一 致的 ${ }^{[9]}$ 。但一步法热处理后的晶粒尺寸较小且结晶 度较低, 会得到三相共存的复相微晶玻璃。

图 4 为经过两步法热处理后样品的 XRD 图谱。 经过 $650 \sim 700^{\circ} \mathrm{C}$ 形核处理后, 析出晶体为 $\mathrm{CaF}_{2}$ 和硬 硅钻石; 随着温度继续升高至 $800^{\circ} \mathrm{C} 、 850^{\circ} \mathrm{C}$ 以及时 间延长, $\mathrm{CaF}_{2}$ 晶核逐渐消失, 硬硅钻石转变为硅碱 钙石或 $\alpha$-硅碱钻石。其中 $\alpha$-硅碱钙石(Frankamenite) 是硅碱䥻石的类似晶体, 它与硅碱钻石的区别在于 着基和 $\mathrm{F}$ 含量的差异, 结构非常类似, 衍射峰也基 本一致 ${ }^{[17]}$, 这里不做过多区分。物相分析结果显示, 两步法充分形核后, 硅碱钻石快速生长, 硬硅钻石 转变为硅碱钻石, 可获得较高的结晶度。

\subsection{SEM 组织结构分析}

为了分析热处理制度对组织结构的影响, 分别 选取 $850^{\circ} \mathrm{C}$ 一步法和两步法析晶处理后样品进行 SEM 结构分析。

图 5 为 $850^{\circ} \mathrm{C}$ 处理后微晶玻璃的 SEM 照片。一 步法处理后如图 5(a)所示, 由于残余玻璃相较多, 经 $\mathrm{HF}$ 腐蚀后, $\mathrm{CaF}_{2}$ 和短柱状/岛状的硬硅钙石晶相 被凸显出来，晶体生长和分布比较均匀，而且玻璃 相和晶相是相互咬合存在的，形成类似硅灰石微晶 玻璃的岛屿状互锁结构。经过两步法处理后，微晶玻 璃的组织结构照片如图 5(b)所示, 为典型板条交错

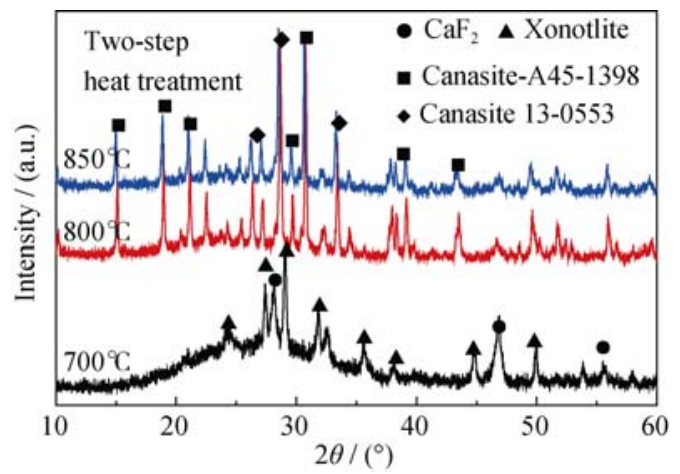

图 4 不同温度两步法热处理后样品的 XRD 衍图谱

Fig. 4 XRD patterns of glass-ceramics after two-step heat treatment

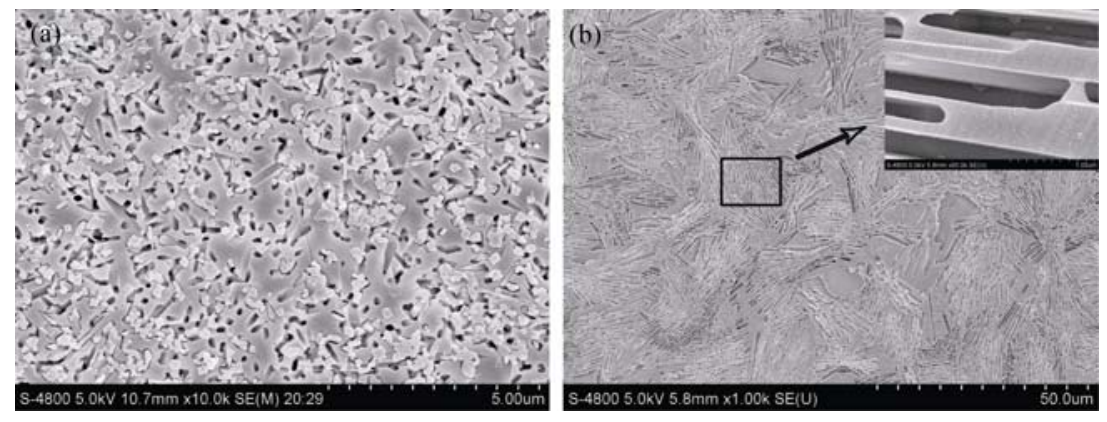

图 $5850^{\circ} \mathrm{C}(\mathrm{a})$ 一步法和(b)两步法热处理后微晶玻璃的微观结构形貌

Fig. 5 SEM images of glass-ceramics after one-step (a) and two-step (b) heat treatment Insert is the enlarged image of the bladed morphology 
状的硅碱钻石集束结构, 放射状的硅碱钻石晶体束 尺寸约为 $30 \mu \mathrm{m}$, 不同取向的晶体团簇区域之间相互 交错, 随机取向, 形成明显的互锁结构, 且晶体间结 合致密完整。图 5(b)插图为交错状板条状硅碱钙石放 大形貌照片, 可见板条宽度小于 $1 \mu \mathrm{m}$, 板条间为残 余玻璃相, 二者形成相互层叠交错结构。这种内含交 错互锁板条状晶体的组织结构, 有理想的晶体解理 面和可能潜在的热膨胀等各向异性, 断裂时能够引 发裂纹扭转增韧和新生微裂纹增韧, 使材料具有较 高的抗弯强度和较高的断裂韧性。

\section{3 力学性能与组织结构的关系}

为了进一步分析不同热处理后组织结构对力学性 能的影响, 分别选取一步法和两步法 $850^{\circ} \mathrm{C}$ 析晶处理后 样品进行力学性能测试, 性能数据如表 1 所示。结果对 比可知, 热处理前后 RCSF 基础玻璃和微晶玻璃硬度 和弹性模量没有太大的变化, 显微硬度介于 7.3 7.5 GPa 之间, 弹性模量介于 73 77 GPa。一般来讲, 材料 硬度的大小主要取决于物质内部结构中原子间结合力 的强弱, 结合力越强, 抵抗外力作用的强度就越大, 硬 度越高。而基础玻璃向晶体转变, 材料内部的键合状态 并没有明显的变化, 仅是结构单元发生重新排列, 即 硅酸盐玻璃的硅氧四面体结构和间隙状态变化很小, 仅是硅氧四面体连接角度的转变和重排, 所以晶体的 析出对材料的本征性质, 如硬度和弹性模量影响不大, 表现为显微硬度和弹性模量对组织结构的不敏感性。
对于组织结构敏感的强度、韧性等力学性能则 随着析晶过程发生明显变化。一步法析晶处理后, 材 料的弯曲强度由 $65 \mathrm{MPa}$ 增大到 $90 \mathrm{MPa}$, 而结晶度更 高、组织结构更复杂的两步法硅碱钙石微晶玻璃的 弯曲强度高达 $119 \mathrm{MPa}$ 。尤其是两步法热处理后得到 的硅碱钻石微晶玻璃, 其断裂韧性由 $0.77 \mathrm{MPa} \cdot \mathrm{m}^{1 / 2}$ 增加到 $1.72 \mathrm{MPa} \cdot \mathrm{m}^{1 / 2}$, 冲击韧性则由 $1.32 \mathrm{~kJ} / \mathrm{m}^{2}$ 增加 到 $6.7 \mathrm{~kJ} / \mathrm{m}^{2}$ 。值得注意的是, 由于一步法和两步法制 备微晶玻璃的晶相类型和微观结构均不同, 二者性 能也有较大的差别, 这进一步说明 RCSF 微晶玻璃的 力学性能受晶相和组织结构的影响是巨大的, 不同 晶相类型和微观结构导致裂纹扩展模式和断裂机制 的变化是力学性能差异的主要原因。

\section{4 裂纹扩展机制及增韧机理研究}

为了进一步研究 RCSF 微晶玻璃裂纹扩展模式 和增韧机理, 实验对压痕形貌以及相应的裂纹扩展 进行了观察分析。

图 6 为 $\mathrm{RCSF}$ 基础玻璃的裂纹扩展和压痕形貌 照片，图中可见在菱形压头的对角线位置有明显的 裂纹扩展，且裂纹沿压痕对角线呈直线状扩展，单 侧裂纹扩展长度超过 $60 \mu \mathrm{m}$, 且裂纹缺口光滑平直, 说明该裂纹为典型玻璃脆性材料的快速扩展裂纹, 这与基础玻璃的韧性较低是对应的。

图 7 是一步法处理后 RCSF 微晶玻璃的裂纹扩 展和压痕形貌照片，与基础玻璃相比裂纹扩展长度

表 1 RCSF 基础玻璃和微晶玻璃的力学性能数据表(GC: Glass-ceramics)

Table 1 Mechanical properties of the RCSF glass and glass-ceramics (GC)

\begin{tabular}{cccccc}
\hline Sample & Hardness, H/GPa & Modulus, E/GPa & Flexural/MPa & $\begin{array}{c}\text { Impact toughness / } \\
\left(\mathrm{kJ} \cdot \mathrm{m}^{-2}\right)\end{array}$ & $\begin{array}{c}\text { Fracture toughness } \\
/\left(\mathrm{MPa} \cdot \mathrm{m}^{1 / 2}\right)\end{array}$ \\
\hline Casting glass & $7.48 \pm 0.21$ & $73.66 \pm 1.78$ & $64 \sim 69$ & 1.32 & $0.77 \pm 0.06$ \\
GC-one-step & $7.32 \pm 0.05$ & $77.12 \pm 1.13$ & $83 \sim 97$ & 2.56 & $1.17 \pm 0.06$ \\
GC-two-step & $7.52 \pm 0.31$ & $77.82 \pm 3.23$ & $109 \sim 119$ & 6.70 & $1.72 \pm 0.45$ \\
\hline
\end{tabular}

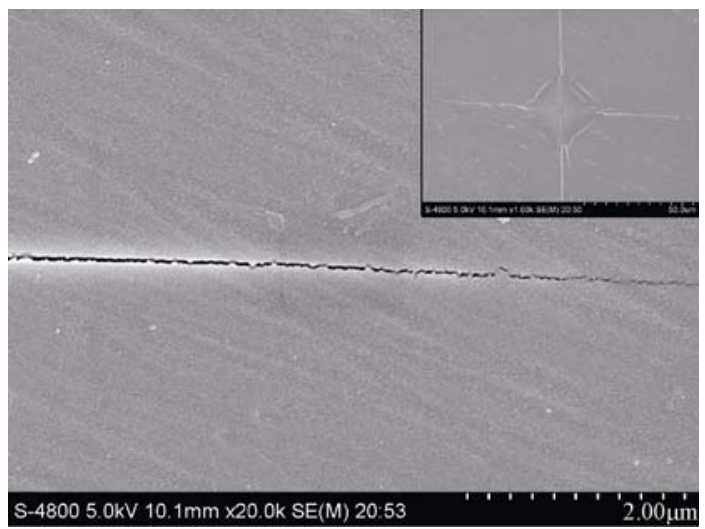

图 $6 \mathrm{RCSF}$ 基础玻璃的裂纹扩展形貌

Fig. 6 SEM images of crack propagation of RCSF casting glasses

Insert shows the morphology of indentation

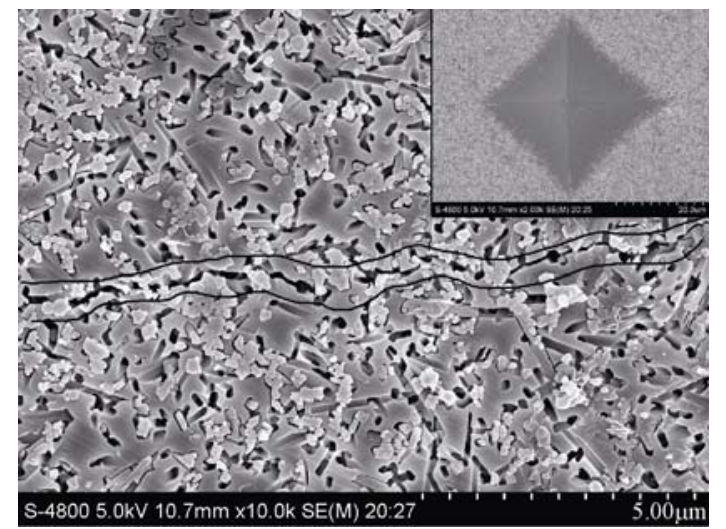

图 7 一步法热处理的 RCSF 微晶玻璃的裂纹扩展形貌

Fig. 7 SEM images of crack propagation of RCSF glassceramics after one-step heat treatment Insert shows the morphology of indentation 
减小至 $20 \mu \mathrm{m}$ 。虽然裂纹仍是沿压痕对角线向外呈 近似直线扩展，但高倍照片显示裂纹扩展受到岛屿 状晶体的影响, 呈现出一个受阻弯曲的断裂路径, 沿晶断裂现象明显。结合上述晶相分析和 SEM 组织 观察分析, 一步法微晶玻璃晶体含量较少, 晶相含 量小于 $50 \mathrm{vol} \%$, 且晶粒尺寸较小 $(0.5 \sim 1.0 \mu \mathrm{m})$ 。故受 到自身组织结构的影响, 一步法热处理获得的微晶 玻璃强度和㓞性提高有限。

图 8 为两步法处理后得到的硅碱钻石微晶玻璃 压痕形貌。硅碱钙石微晶玻璃的压痕形貌与前述完 全不同，四个压痕周围均出现了多条随机取向的裂 纹，在压痕周围不同位置和角度均有不同大小的裂 纹扩展，单条裂纹呈现明显的折线状或台阶状，且 裂纹长度小于 $20 \mu \mathrm{m}$ 。如前所述, 两步法处理后硅 碱钙石微晶玻璃呈板条状交错的硅碱钙石结构, 即 微观组织为放射状硅碱钻石集束, 集束由片状硅碱 钙石聚集而成，片层间为残余玻璃相，其中片状晶 体的长径比在 10 以上。由于每次压痕位置不同, 受 其周围晶体取向的影响，压痕周围裂纹的形成和扩 展呈随机变化。

图 9 为两步法处理后硅碱钻石微晶玻璃裂纹扩 展形貌与组织结构的对照图, 结果显示该硅碱钻石 微晶玻璃的裂纹扩展与其独特的组织结构有密切关 系，根据预制压痕裂纹位置的不同，呈现出不同的 裂纹扩展方式。图 9(a)中预制裂纹扩展路径与板条 状晶体平行, 在尖端应力作用下裂纹沿板条晶体与
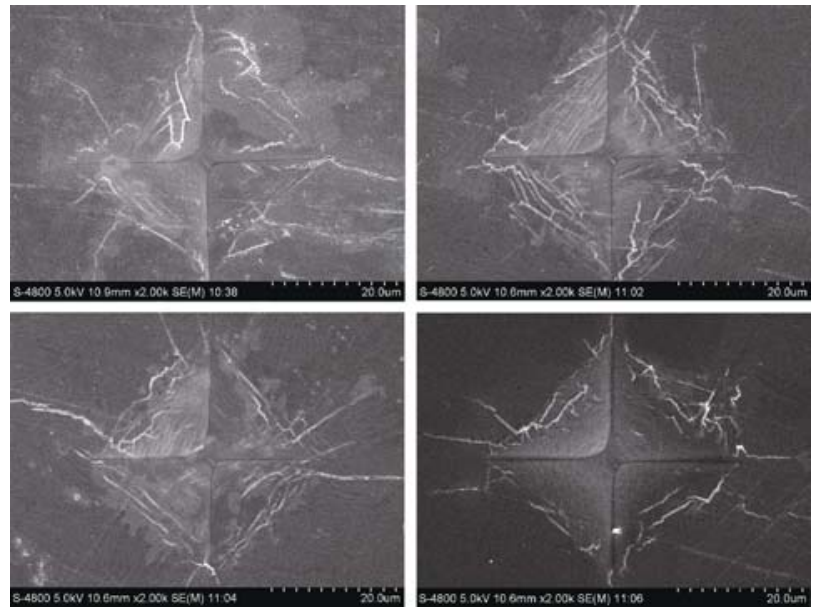

图 8 两步法处理的 RCSF 硅碱钲石微晶玻璃的压痕 SEM 照片 Fig. 8 SEM images of indentation of RCSF canasite glassceramics after two-step heat treatment

玻璃相界面快速平直扩展，即存在明显的解理型脆 性断裂。图 9(b、c)为不同取向晶体集束交界处折线 状或台阶状的裂纹形貌。图 9(b)中穿晶裂纹扩展至 集束界面后，转向沿玻璃相界面解理扩展至垂直取 向的下一晶体, 由于此晶体长径比较大, 裂纹无法 继续偏转，而选择穿过晶体；图 9(c)中沿晶裂纹扩 展至团簇界面后，由于扩展方向与下一片状晶体取 向角度较小, 裂纹则选择能量最小的界面偏转后继 续行进。图 9(d)中裂纹横跨两个不同取向的集束, 均为穿晶断裂, 这种裂纹在行进过程中, 由于偏转 角太大或偏转后行程过长使得扩展阻力和扩展功增
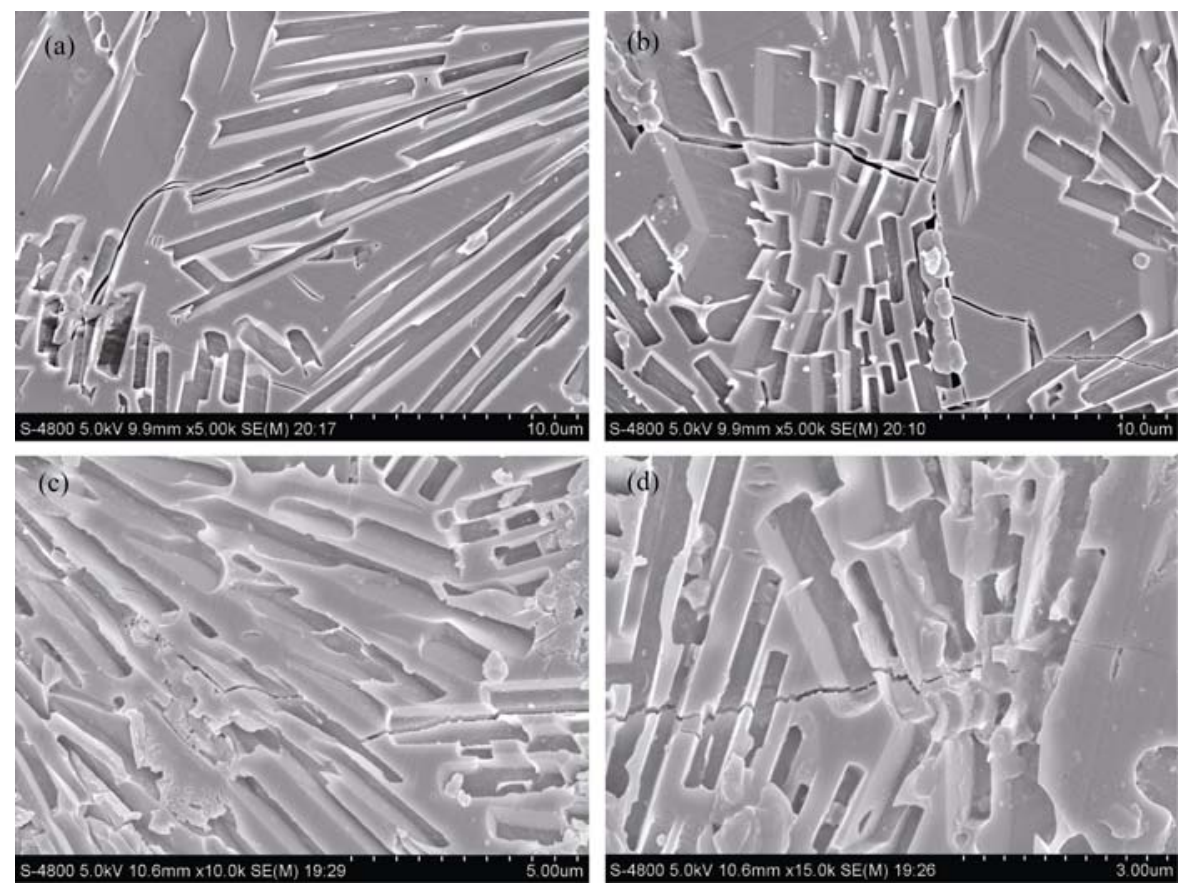

图 9 两步法处理的 RCSF 硅碱钻石微晶玻璃的裂纹扩展 SEM 照片

Fig. 9 SEM images of crack propagation of RCSF canasite glass-ceramics after two-step heat treatment 
加, 而裂纹总是选择消耗最小的路径扩展, 故出现 了板条状晶体的横向穿晶断裂。上述现象表明在硅 碱钙石微晶玻璃中, 具有一定的长径比的板条晶体 (大于临界长径比) 能够起到很好的桥联作用, 使得 裂纹无法绕过纤维或板条而发生快速断裂, 进而起 到承载补强作用。综上所述, 硅碱钙石微晶玻璃中 存在穿晶断裂和沿晶断裂两种裂纹扩展模式, 其独 特的板条交错结构使得裂纹呈曲折路径进行, 进而 其宏观断口呈现出类似脱粘纤维的粗糙界面。

上述通过裂纹偏转、弯曲而消耗额外的能量, 均属于非屏蔽增韧。此外, 在微晶玻璃中有大量第 二相晶体析出, 会因第二相颗粒与基体间弹性模量 和热膨胀系数的失配引入残余应力, 进而起到一定 的残余应力增㓞补强作用。图 10 测定了 RCSF 玻璃 析晶前后基础玻璃和硅碱钙石微晶玻璃的热膨胀曲 线, 析晶处理后材料线膨胀系数由 $94 \times 10^{-7} / \mathrm{K}$ 降低 到 $83.6 \times 10^{-7} / \mathrm{K}$, 即硅碱钻石晶体的膨胀系数稍小。 这种情况微晶玻璃中垂直于玻璃与颗粒界面为拉应 力, 平行于界面为压应力。裂纹扩展总是沿应力场 的薄弱环节进行, 即平行于压应力轴, 垂直于张应 力轴。上述的沿晶裂纹在沿着界面扩展时, 裂纹行 进过程就需要消耗更多的能量。这种由两相性质差 异带来的内应力与裂纹尖端应力相互作用, 有利于 应力集中缓和甚至增加裂纹偏转的几率, 可认为存 在屏蔽增韧机制。

综上所述, 以板条交错状硅碱钙石为主晶相的 微晶玻璃具有良好的力学性能, 尤其是较高的强度 和韧性，这是各种增强和增韧作用机制的综合效 应。虽然本文中的板条状球晶已经表现出类似纤维 状晶体的良好增韧效果, 但是和文献[12]中材料优 异的纤维/针状交错结构相比仍有一定的差距。因此, 今后可通过进一步改变材料组织结构, 提高结晶度, 在长径比一定的情况下减小晶体集束大小, 增加板

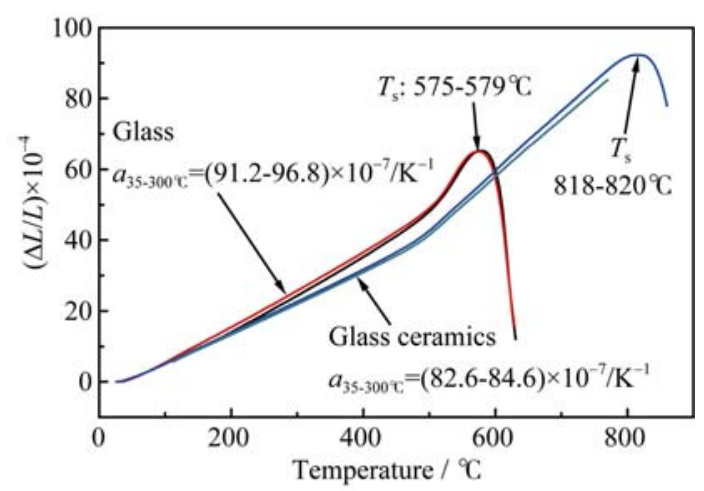

图 $10 \mathrm{RCSF}$ 玻璃析晶前后的热膨胀曲线

Fig. 10 Thermal expansion curves of casting glass and canasite glass-ceramics
条晶体取向混乱度, 减少沿晶断裂的裂纹数量及裂 纹长度, 进一步提高该体系微晶玻璃的力学性能。

\section{3 结论}

通过改变热处理制度制备了两种不同显微结构 的硅碱钙石微晶玻璃，并对该微晶玻璃的析晶过程、 组织结构和力学性能进行研究, 重点讨论了以硅碱 钙石为主晶相微晶玻璃的裂纹扩展机制及其增韧机 理, 主要结论如下:

1) RCSF 基础玻璃在热处理时首先析出 $\mathrm{CaF}_{2}$ 微 晶作为异质晶核, 形成以硬硅钙石/硅碱钻石为主晶 相的微晶玻璃，两步法处理后可获得板条交错的硅 碱钙石结构;

2) 板条交错硅碱钙石晶相具有明显的增强和增 韧效果, 该硅碱钙石微晶玻璃具有优异的力学性能, 其弯曲强度高达 $119 \mathrm{MPa}$ ，断裂韧性为 $1.72 \mathrm{MPa} \cdot \mathrm{m}^{1 / 2}$, 冲击韧性可达 $6.7 \mathrm{~kJ} / \mathrm{m}^{2}$;

3) 硅碱钻石微晶玻璃裂纹的穿晶和沿晶扩展受 周围板条晶体取向及晶界的影响，裂纹呈随机取向 的折线状或台阶状; 在一定长径比尺度下的板条状 硅碱钙石晶体具有较好的桥联作用，承载补强效果 显著; 此外基础玻璃与晶体间的残余应力还具有一 定的屏蔽增韧效果。

\section{参考文献:}

[1] CHENG JINSHU, LI HONG, TANG LI YING, et al. Glassceramics. Chemical Industry Press, 2006.

[2] HOLAND W, BEALL G H. Glass-ceramic Technology. The Ameriean Ceramie Society, Westerville, OH, 2002.

[3] BEALL G H. Alkali Metal, Calcium Fluorosilicate Glass-ceramic Articles. US Patent No. 4386162, 1983.

[4] BEALL G H. Chain silicate glass-ceramics. Journal of Non-Crystalline Solids, 1991, 129(1/2/3):163-173.

[5] WANG SHUANGHUA, YANG ZHIFANG, YANG ZHI-HONG, et al. Preparation and machinable properties of the in-situ fluorcanasite micro glass-ceramic composite. Materials Review, 2007, 21(9): 96-99.

[6] MILLER C A, KOKUBO T, REANEY I M, et al. Formation of apatite layers on modified canasite glass-ceramics in simulated body fluid. Journal of Biomedical Materials Research, 2002, 59(3): 473-480.

[7] BARROS VMD, SALATA L A, SVERZUT C E, et al. In vivo bone tissue response to a canasite glass-ceramic. Biomaterials, 2002, 23(14): 2895-2900.

[8] WANG CHUNHUA, YANG XIAOJUN. Study on the structure 
and properties of low-temperature glass-ceramic bond. Diamond \& Abrasives Engineering, 2011, 2(31): 58-62.

[9] KANCHANARAT N, Miller C A, HATTON P V, et al. Early stages of crystallization in canasite-based glass ceramics. Journal of the American Ceramic Society, 2005, 88(11): 3198-3204.

[10] HILL R G, LAW R V, O’DONNELL M D, et al. Characterisation of fluorine containing glasses and glass-ceramics by $19 \mathrm{~F}$ magic angle spinning nuclear magnetic resonance spectroscopy. Journal of the European Ceramic Society, 2009, 29(11): 2185-2191.

[11] MIRSANEH M, REANEY I M, JAMES P F, et al. Effect of $\mathrm{CaF}_{2}$ and $\mathrm{CaO}$ substituted for $\mathrm{MgO}$ on the phase evolution and mechanical properties of K-Fluorrichterite glass ceramics. Journal of the American Ceramic Society, 2006, 89(2): 587-595.

[12] JOHNSON A, SHAREEF M Y, VAN NOORT R, et al. Effect of furnace type and ceramming heat treatment conditions on the biaxial flexural strength of a canasite glass-ceramic. Dental Materials, 2000, 16(4): 280-284.

[13] JOHNSON A, SHAREEF M Y, WALSH J M, et al. The effect of casting conditions on the biaxial flexural strength of glass-ceramic materials. Dental Materials, 1998, 14(6): 412-416.

[14] SONG SHUXIN, ZHENG WEIHONG. Effect of CaO content on structure and crystallization of RCSF glass-ceramics. Journal of Wuhan University of Technology, 2009, 31(22): 23-26.

[15] CHENG JINSHU, DENG WEI, ZHENG WEIHONG, et al. Effect of $\mathrm{K}_{2} \mathrm{O}$ content on crystallization of $\mathrm{R}_{2} \mathrm{O}-\mathrm{CaO}-\mathrm{SiO}_{2}-\mathrm{F}$ glass-ceramics. Journal of Wuhan University of Technology, 2010, 32(8): 26-29.

[16] SONG SHUXIN, CHENG JINSHU, ZHENG WEIHONG, et al. Effect of mass fraction of $\mathrm{Al}_{2} \mathrm{O}_{3}$ on structure and crystallization of
$\mathrm{R}_{2} \mathrm{O}-\mathrm{CaO}-\mathrm{SiO}_{2}-\mathrm{F}$ glass-ceramics. Glass \& Ename, 2009, 37(4): 5-9.

[17] ZHENG WEIHONG, YANG KUN. Effect of $F$ on phase transition of $\mathrm{R}_{2} \mathrm{O}-\mathrm{CaO}-\mathrm{SiO}_{2}$ system glass-ceramics. Journal of Wuhan University of Technology, 2010, 32(22): 84-86.

[18] DENG WEI, SONG SHUXIN. Effect of F content on structure and crystallization of RCSF glass-ceramics. Journal of Wuhan University of Technology, 2009, 31(22): 16-18.

[19] ZHOU XIYA, HU JUN, ZHOU BINYANG, et al. Effect of fluorine content on crystallization of $\mathrm{CaO}-\mathrm{SiO}_{2}-\mathrm{R}_{2} \mathrm{O}-\mathrm{F}$ glass-ceramic. Bulletin of the Chinese Ceramic Society, 2009, 28(5): 168-172.

[20] DENG WEI, GONG YUXUAN, CHENG JIN-SAU, et al. Liquidphase separation and crystallization of high silicon canasite-based glass ceramic. Journal of Non-Crystalline Solids, 2014, 385: $47-54$.

[21] CHENG JINSHU, DENG WEI, ZHENG WEIHONG, et al. Phase transition of different crystals in canasite-based glass-ceramics. Journal of the Chinese Ceramic Society, 2012, 40(10): 1415-1420.

[22] LI HONG, WU YANPING, CHEN FANGWEI, et al. Influence of Heat Treatment on crystallization properties of colored RCS glass-ceramics. Journal of the Chinese Ceramic Society, 2011, 39(1): 143-147.

[23] YANG LANLAN, DENG ZHENLU, DENG WEI, et al. Influence of $\mathrm{Co}_{2} \mathrm{O}_{3}$ and $\mathrm{K}_{2} \mathrm{Cr}_{2} \mathrm{O}_{7}$ on colouring and crystallization of $\mathrm{R}_{2} \mathrm{O}-\mathrm{CaO}-\mathrm{SiO}_{2}-\mathrm{F}$ glass-ceramics. Journal of Wuhan University of Technology, 2010, 32(22): 32-35.

[24] DENG WEI, CHENG JINSHU, TAN PEIJING, et al. Chemical durability and weathering resistance of canasite based glass and glass-ceramics. Journal of Non-Crystalline Solids, 2012, 358(21): 2847-2854. 